\title{
POSITIVE DEFINITE FUNCTIONS AND VOLTERRA INTEGRAL EQUATIONS ${ }^{1}$
}

\author{
BY JAMES S. W. WONG
}

Communicated by Richard Goldberg, January 3, 1974

1. Introduction. The purpose of this research announcement is to describe a new approach for studying asymptotic behavior of solutions of functional equations involving a Volterra operator. More specifically, we study the role played by positive definite and related classes of functions as convolution kernels of the Volterra operators.

2. Positive and $D$-positive definite functions. Let $a(t) \in C(0, \infty) \cap$ $L^{1}(0,1)$. We say that $a(t)$ is positive definite if for any function $\varphi(t) \in$ $C[0, \infty)$, the quadratic form

$$
Q_{a}[\varphi](T)=\int_{0}^{T} \varphi(t) \int_{0}^{t} a(t-\tau) \varphi(\tau) d \tau d t \geqq 0, \quad T \geqq 0 .
$$

Similarly, we say that $a(t)$ is $D$-positive definite if the quadratic form

$$
R_{a}[\varphi](T)=\int_{0}^{T} \varphi(t) \frac{d}{d t} \int_{0}^{t} a(t-\tau) \varphi(\tau) d \tau d t \geqq 0, \quad T \geqq 0 .
$$

This definition of positive definite functions differs slightly from that of Bochner since $a\left(0_{+}\right)$is not assumed to exist and remains finite. The present form, as applied to the study of Volterra integral equations, was first introduced by Halanay [1], although he assumed that $a(t) \in C[0, \infty)$, thereby excluding the interesting case $t^{-v}, 0<v<1$, the "so-called" Abel kernels. The idea of $D$-positive definite functions may be found in MacCamy [6] although his definition on $a(t)$ is even more restrictive. There is some ambiguity as to what $R_{a}[\varphi](T)$ means when $a\left(0_{+}\right)$does not exist. This

AMS (MOS) subject classifications (1970). Primary 45D05, 45M10; Secondary 35B35, 35B40.

Key words and phrases. Positive definite, Fourier transforms, Volterra integral equation, partial differential-functional equation, stability, asymptotic behavior, viscoelasticity.

${ }^{1}$ Research supported in part by Army Research Office, Durham, through Contract No. DA-ARO-D-31-124-72-G95 
difficulty is overcome by first restricting $R_{a}$ to functions in $C^{1}[0, \infty)$ and then extending to all of $C[0, \infty)$ by passing to the limit.

A special subclass of positive definite functions are the negative exponentials, $\varepsilon e^{-\alpha t}, \varepsilon, \alpha>0$, in terms of which we define a larger subclass called strongly positive definite functions. We call the function $a(t)$ strongly positive definite, if there exists $\varepsilon, \alpha>0$ such that $a(t)-\varepsilon e^{-\alpha t}$ is positive definite. On the other hand, in view of Corollary (b) below, we can consider the special subclass of $D$-positive definite functions which are nonnegative, nonincreasing, and do not belong to $L^{1}(0, \infty)$. Denote this special class by $\mathscr{L}$. We define $a(t)$ to be strongly $D$-positive definite if there exists $b(t) \in \mathscr{L}$ such that $a(t)-b(t)$ is $D$-positive definite.

Criteria for positive and $D$-positive definiteness of $a(t)$ can be given in terms of its Laplace transform $\hat{a}(s)$ defined by

$$
\hat{a}(s)=\lim _{T \rightarrow \infty} \int_{0}^{T} e^{-s t} a(t) d t .
$$

We now assume in addition that $a(t) \in L^{\infty}(0, \infty)$ (more precisely, it is sufficient to assume that $a(t)$ belongs to the space of tempered distributions).

THEOREM 1. Suppose that $\hat{a}(i \omega)$ exists almost everywhere for $\omega \in \boldsymbol{R}$. Then,

(a) $\operatorname{Re} \hat{a}(i \omega) \geqq 0$ a.e. $\Rightarrow a(t)$ is positive definite, and

(b) $\operatorname{Im} \omega \hat{a}(i \omega) \leqq 0$ a.e. $\Rightarrow a(t)$ is $D$-positive definite.

Using Theorem 1, one can now formulate similar criteria for strongly positive and $D$-positive definite functions. As a consequence of this, we have

COROLlaRY. (a) Let a(t) be nonnegative, $a^{\prime}(t)$ nonpositive and nondecreasing, then $a(t)$ is positive definite. If, in addition, $a^{\prime}(t) \not \equiv 0$ then $a(t)$ is strongly positive definite.

(b) Let $a(t)$ be nonnegative and nonincreasing, then $a(t)$ is $D$-positive definite.

Using these results, one can show that any trigonometric polynomials in cosines with positive coefficients are positive definite, whilst trigonometric polynomials in sines with positive coefficients are $D$-positive definite. The important class of Abel kernels, $t^{-v}, 0<v<1$, are both strongly positive and $D$-positive definite. Furthermore, some weakly singular kernels involving logarithms can also be shown to be strongly positive and strongly $D$-positive definite. For proofs of these results refer to [7], [8], [9]. 
3. Volterra integral equations. We consider the following two nonlinear Volterra integral equations studied by Levin [3], [4]:

$$
\begin{aligned}
u^{\prime}(t) & =f(t)+\int_{0}^{t} a(t-\tau) g(u(\tau)) d \tau, \\
u(t) & =f(t)+\int_{0}^{t} a(t-\tau) g(u(\tau)) d \tau,
\end{aligned}
$$

where $f(t) \in L^{1}(0, \infty)$ and $g(u)$ satisfies

$$
\begin{aligned}
& g(u) \in C(-\infty, \infty), \quad u g(u)>0, \quad u \neq 0 ; \\
& \lim _{|u| \rightarrow \infty} G(u)=\infty, \quad|g(u)| \leqq M(1+G(u)), \quad G(u)=\int_{0}^{u} g(\xi) d \xi .
\end{aligned}
$$

It is known [2] that if $a(t)$ is nonnegative, $a^{\prime}(t)$ nonpositive and nondecreasing, then all solutions of (3) are bounded, and if, in addition, $a^{\prime}(t) \not \equiv 0$, then all solutions tend to zero. On the other hand, if $a(t)$ is nonnegative, nonincreasing, then all solutions of (4) are bounded, and if, in addition, $a(t) \notin L^{1}(0, \infty)$, then all solutions tend to zero [5]. Using the results given in $\S 1$, we can now state (see [9])

TheOREM 2. If $a(t)$ is positive and D-positive definite, respectively, then all solutions of (3) and (4) are bounded, respectively. Moreover, if $a(t)$ is strongly positive and $D$-positive definite, respectively, then all solutions of (3) and (4) tend to zero, respectively.

4. An integro-partial differential equation. The concepts of positive and $D$-positive definiteness can also be used to study asymptotic behavior of solutions of integro-partial differential equations with equal efficiency. We consider the following initial boundary value problem which arises from the study of viscoelasticity:

$$
\begin{aligned}
\frac{\partial u}{\partial t}(x, t) & =\int_{0}^{t} a(t-\tau)\{\Delta u(x, \tau)+g(\tau, u(x, \tau))\} d \tau, \quad x \in \Omega, t \geqq 0, \\
u(x, t) & =0, \quad x \in \partial \Omega \text { and } u(x, 0)=u_{0}(x),
\end{aligned}
$$

where $\Omega$ is a bounded domain in $\boldsymbol{R}^{n}$ and $g(t, u)$ is a nonlinear perturbing term satisfying

$$
|g(t, u)| \leqq \lambda(t)|u|^{\sigma}, \quad 0 \leqq \sigma \leqq 1, \lambda(t) \in L^{1}(0, \infty) .
$$

Here we are interested in establishing the existence of solutions of (5) and the asymptotic behavior of these solutions as $t \rightarrow \infty$. Denote by $\rho(t)$ the creep compliance function corresponding to $a(t)$, i.e. $\int_{0}^{t} \rho(t-\tau) a(t) d \tau=t$. 
THEOREM 3. Let $p^{\prime \prime}(t)$ be positive definite and $\lambda(t) \in L^{2}(0, T)$, for every finite $T>0$. Then equation (5) has a generalized solution $u(x, t)$ in the sense that $u(x, t) \in L^{2}\left(0, T ; H_{0}^{1}(\Omega)\right), \partial u(x, t) / \partial t \in L^{2}\left(0, T ; L^{2}(\Omega)\right)$ and satisfies (5) weakly in $L^{2}(\Omega)$. If, in addition, a(t) is strongly positive and $g_{u}(t, u)$ is bounded, then

$$
\lim _{t \rightarrow \infty}\|u(\cdot, t)\|_{1}=0
$$

where \|\|$_{1}$ denotes the norm for $H_{0}^{1}(\Omega)$.

The hypotheses required on $a(t)$ and $\rho(t)$ are easily satisfied if $a(t)=$ $\varepsilon e^{-\alpha t}, \varepsilon, \alpha>0$. For other examples, we refer the reader to [10]. The proof of existence is based upon the Galerkin method and Sobolev's embedding lemma, whereas the asymptotic behavior is derived using Gårding's inequality and a priori estimates for elliptic partial differential operators. Details of these results together with extensions of positive and $D$-positive definite functions to Hilbert spaces will appear elsewhere [10].

\section{REFERENCES}

1. A. Halanay, On the asymptotic behavior of the solutions of an integro-differential equation, J. Math. Anal. Appl. 10 (1965), 319-324. MR 31 \#579.

2. K. B. Hannsgen, On a nonlinear Volterra equation, Michigan Math. J. 16 (1969), 365-376. MR 40 \#3225.

3. J. J. Levin, The asymptotic behavior of the solution of a Volterra equation, Proc. Amer. Math. Soc. 14 (1963), 534-541. MR 27 \#2824.

4. - The qualitative behavior of a nonlinear Volterra equation, Proc. Amer. Math. Soc. 16 (1965), 711-718. MR 32 \#8081.

5. S.-O. London, On a nonlinear Volterra integral equation, J. Differential Equations 14 (1973), 106-120.

6. R. C. MacCamy, Nonlinear Volterra equations on a Hilbert space, J. Differential Equations (to appear).

7. R. C. MacCamy and J. S. W. Wong, Stability theorems for some functional equations, Trans. Amer. Math. Soc. 164 (1972), 1-37. MR 45 \#2432.

8. J. S. W. Wong and R. Wong, Asymptotic solutions of linear Volterra equations with singular kernels, Trans. Amer. Math. Soc. (to appear).

9. J. S. W. Wong, The role of positive definite functions in the study of Volterra integral equations, Proc. USC Conference (to appear).

10. - A nonlinear integro-partial differential equation arising from viscoelasticity (to appear).

Department of Mathematics, University of lowa, Iowa City, Iowa 52242 